Hector Zambrano, MD, MSC

National Secretary of Sience and Technology

(SENESCYT)

National Institute for Research in Public Health (INSPI)

Guayaquil

Guayas

Ecuador

Martha Montalvan, MD

Academic Coordinator

Faculty of Medicina Universidad Espiritu

Santo Guayaquil-Samborondon

Ecuador

E-mail: martham@uees.edu.ec

Jimmy Cabezas, MD

Universidad Espiritu Santo

Guayaquil-Samborondon

Ecuador

Yin $\mathrm{Lu}, \mathrm{PhD}$

Catherine S. Yang, MD

Leonard M. Milstone, MD

Department of Dermatology

Yale University

\section{Melanoma: when dermoscopy is a pitfall}

A growing body of evidence on dermoscopy has documented its value for an efficient approach toward pigmented skin lesions, increasing diagnostic accuracy and avoiding unnecessary procedures. However, melanoma can mimic seborrheic keratosis (SK), either clinically or by dermoscopy. We report a striking case of a large and invasive melanoma simulating SK on both clinical and dermoscopic examinations.

A 72-year-old woman presented with a longstanding lesion on her left thigh (Fig. Ia). The patient noticed change in the last six months, namely enlargement and pruritus. Clinical examination revealed a $20 \times$ II $\mathrm{mm}$ brownish papillomatous plaque with a central darker area. Dermoscopy (Fig. Ib) revealed a well-circumscribed, raised, oval lesion. There were several shades of brown and a black irregular, area with some comedo-like openings and a less pigmented keratotic plug in the center. Its surface was irregular and the presence of fissures and ridges formed a cerebriform appearance. Multiple milia-like cysts and dotted vessels with a whitish halo were easily found. A careful observation also disclosed linear irregular vessels, and some globular structures were asymmetrically distributed at the periphery of the lesion. The lesion was excised and proved a $2.7 \mathrm{I} \mathrm{mm}$ thick melanoma with epidermal hyperplasia, hyperkeratosis, and some horn pseudocysts.
New Haven

CT

USA

Dr Keith Choate, $\mathrm{MD}, \mathrm{PhD}$

Department of Dermatology

Yale University School of Medicine

333 Cedar Street, New Haven

CT o65Io

USA

E-mail: keith.choate@yale.edu

Conflict of interest: All the authors declare there is no conflict of interest.

\section{References}

I Fischer J. Autosomal recessive congenital ichthyosis.

J Invest Dermatol 2009; I29: I3I9-I32I.

2 Akiyama M, Takizawa Y, Kokaji T, et al. Novel mutations

of TGMI in a child with congenital ichthyosiform

erythroderma. Br J Dermatol 200I; I44: 40I-407.

3 Herman ML, Farasat S, Steinbach PJ, et al.

Transglutaminase-I gene mutations in autosomal recessive congenital ichthyosis: summary of mutations (including 23 novel) and modeling of TGase-I. Hum Mutat 2009; 30:

$537-547$.

Wide local excision was performed. Staging included a thoraco-abdomino-pelvic computed tomography (normal) and sentinel lymph node biopsy (negative). Assigned stage IIb $\left(\mathrm{T}_{3} \mathrm{bNoMo}\right.$ ), the patient remains in regular follow-up, with no recurrence.

Comedo-like openings and milia-like cysts correspond to the presence of horn pseudocysts at different levels of the epidermis and are easily found on microscopy (Fig. Ic). They are the dermoscopic hallmarks of SK and are observed, respectively, in $7 \mathrm{I}$ and $66 \%$ of pigmented SK. ${ }^{\mathrm{I}}$ Owing to their superficial location, they can sometimes conceal the recognition of other more specific dermoscopic features. They are not pathognomonic of SK and may be seen in papillomatous melanocytic nevi or even in melanoma. ${ }^{2,3}$ In our case, we hypothesized a nevoid origin to the melanoma, supported by histological examination.

The prevalence of melanoma in lesions submitted for histological examination, clinically resembling SK, has been estimated to be $0.66 \% .^{4}$ Difficult melanomas remain a serious concern even in the dermoscopy era and include early lesions, featureless tumors, and melanomas mimicking nevi or other benign lesions. ${ }^{5,6}$ Current strategies to further increase our diagnostic performance include serial examinations with digital dermoscopy, non-invasive evaluation with confocal microscopy and an effective dermoscopy-pathology correlation. 

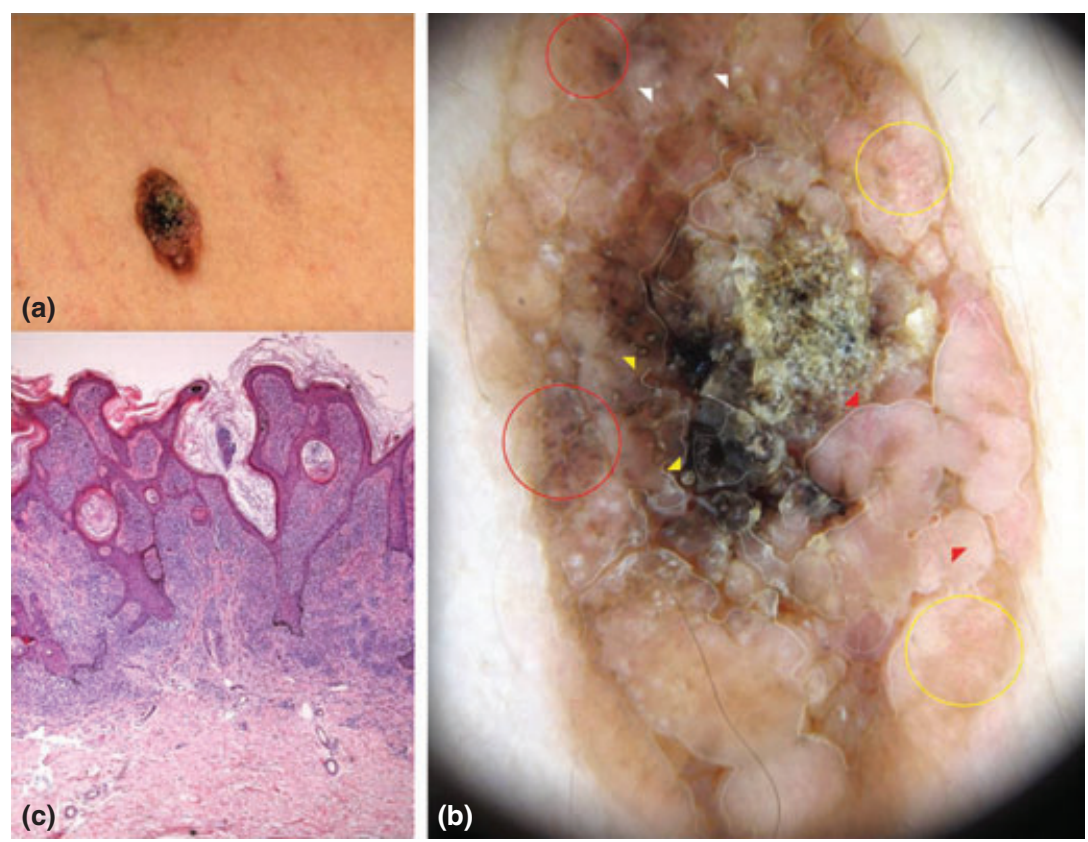

Figure 1 (a) Clinical image. (b) Dermoscopic examination: comedo-like openings (yellow arrowheads), multiple milia-like cysts (white arrowheads), dotted vessels with a whitish halo (yellow circle), linear irregular vessels (red arrowhead), globular structures at the periphery of the lesion (red circles) (see text for fuller details). (c) Melanoma showing marked epidermal hyperplasia and hyperkeratosis with horn pseudocysts (hematoxylin and eosin, original magnification $\times 50$ )

A known cognitive error in perception, termed search satisfaction, commonly occurs in radiology, as well in dermatology, when a physician stops searching once he finds a positive result. ${ }^{7}$ In this case, subtle clinical clues that would raise suspicion included the large size of a solitary lesion in the anterior thigh, which is not the usual scenario for SK. Furthermore, careful dermoscopic examination could reveal discrete linear irregular vessels, which are always a worrisome feature, and the irregularly sized and distributed globular structures as a helpful clue to accurately identify the melanocytic nature of this tumor. As a final note, it is always of utmost importance that clinicians keep a high index of suspicion for malignancy to avoid misdiagnoses and adverse outcomes.

Vera Teixeira, MD

David Serra, MD

Department of Dermatology

Coimbra University Hospital

Coimbra

Portugal

Ricardo Vieira, MD

Américo Figueiredo, PhD, MD

Department of Dermatology

Coimbra University Hospital

Faculty of Medicine
University of Coimbra

Coimbra

Portugal

Maria José Julião, MD

Department of Pathology

Coimbra University Hospital

Coimbra

Portugal

Vera Teixeira, MD

Serviço de Dermatologia

Hospitais da Universidade de Coimbra

Praceta Mota Pinto

3000-075 Coimbra

Portugal

E-mail: vera.teixeira.derm@gmail.com

Conflicts of interest: None.

\section{References}

I Braun RP, Rabinovitz HS, Krischer J, et al. Dermoscopy of pigmented seborrheic keratosis: a morphological study. Arch Dermatol 2002; I38: I 556-I 560.

2 Argenziano G, Rossiello L, Scalvenzi M, et al. Melanoma simulating seborrheic keratosis: a major dermoscopy pitfall. Arch Dermatol 2003; I39: 389-391. 
3 Carrera C, Segura S, Palou J, et al. Seborrheic keratosis like melanoma with folliculotropism. Arch Dermatol 2007; I43: 373-376.

4 Izikson L, Sober AJ, Mihm MC Jr, et al. Prevalence of melanoma clinically resembling seborrheic keratosis: analysis of 9204 cases. Arch Dermatol 2002; I38: I 562-I 565.

5 Puig S, Argenziano G, Zalaudek I, et al. Melanomas that failed dermoscopic detection: a combined

\section{Ulcerative sarcoidosis}

Sarcoidosis is a chronic granulomatous multisystem disease of unknown origin that can affect any organ of the body. Skin lesions are relatively common and are classi-

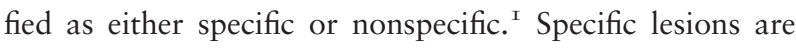
those that show typical sarcoid granulomas, including lupus pernio, infiltrated plaques, maculopapular eruptions, subcutaneous nodules, and infiltration of old scars. ${ }^{\mathrm{I}}$ The nonspecific skin lesion includes erythrodermic, ichthyosiform, psoriasiform, verrucous, or ulcerated sarcoidosis. We report a typical ulcerative sarcoidosis case with characteristic pulmonary lesions.

A 52-year-old woman presented with an 8-year history of plaque on her face and I-year history of nodules and ulcers on her back, chest, and extremities (Fig. Ia,b). Skin biopsy from both nodules and ulcers showed diffuse nonnecrotizing granulomatous inflammation with numerous multinucleated giant cells, lymphocytes, epithelioid cells, and plasma cell infiltration (Figs. Ic and 2). The chest computed tomography scan showed bilateral pulmonary

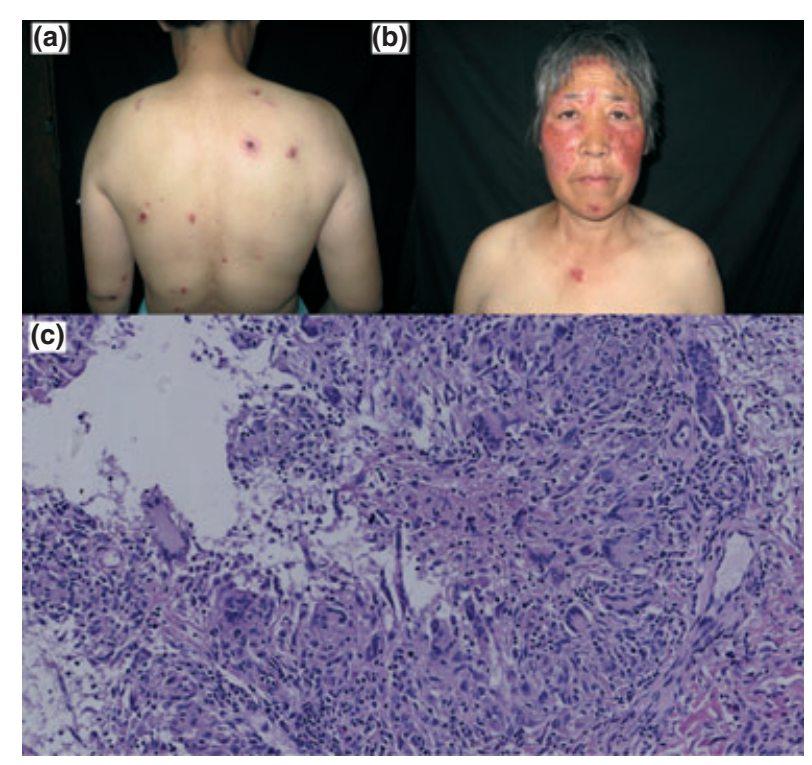

Figure 1 (a) Nodules and ulcers on her back, chest, and extremities. (b) Plaque on her face. (c) Skin biopsy showed diffuse non-necrotizing granulomatous inflammation with numerous multinucleated giant cell, lymphocytes, epithelioid cells, and plasma cells infiltration (hematoxylin-eosin $\times 200$ ) clinicodermoscopic approach for not missing melanoma. Dermatol Surg 2007; 33: I 262-I 273.

6 Pizzichetta MA, Stanganelli I, Bono R, et al. Dermoscopic features of difficult melanoma. Dermatol Surg 2007; 33: 9I-99.

7 Braga JC, Scope A, Klaz I, et al. Melanoma mimicking seborrheic keratosis: an error of perception precluding correct dermoscopic diagnosis. J Am Acad Dermatol 2008; 58: $875^{-880 .}$

nodules with peribronchovascular micronodules and enlarged mediastinal lymph nodes. Bronchoalveolar lavage excluded infection, particularly tuberculosis. Angiotensin-converting enzyme was elevated at Ioo U/l (normal o-56). The findings were consistent with sarcoidosis. Tuberculosis, cryptococcosis, histoplasmosis, and other infective diseases were excluded by the laboratory analysis. Therefore, steroid therapy was initiated and the patient placed on $60 \mathrm{mg}$ prednisone daily. Under this treatment, the sarcoidosis manifestations improved generally, and the prednisone dose was subsequently reduced.

Sarcoidosis is a chronic multisystem disease of unknown origin involving the eyes, skin, lungs, heart, bones, and nerves. Histologically, sarcoidosis is characterized by the formation of noncaseating granulomas. The most common manifestation of the disease is the intrathoracal combination of bilateral hilar lymphadenopathy and pulmonary parenchymal involvement. ${ }^{\mathrm{I}}$ The mediastinal and peripheral lymph nodes are reported to be affected in 50-90\% of the patients, the lungs in more than 50\%. ${ }^{2}$ Specific cutaneous manifestations are also common and occur in 9-37\% of the patients. ${ }^{\mathrm{I}}$ These lesions histologically show the noncaseating sarcoid granulomas.

Ulcerative skin lesions rarely occur in sarcoidosis. They have been reported for only I \% of Caucasian patients with sarcoidosis. ${ }^{3}$ Of the 35 patients reported by Albertini's review, ${ }^{3}$ I 8 were African-American, II were Caucasian, and one was Japanese. Yoo et al. ${ }^{4}$ reported seven cases of

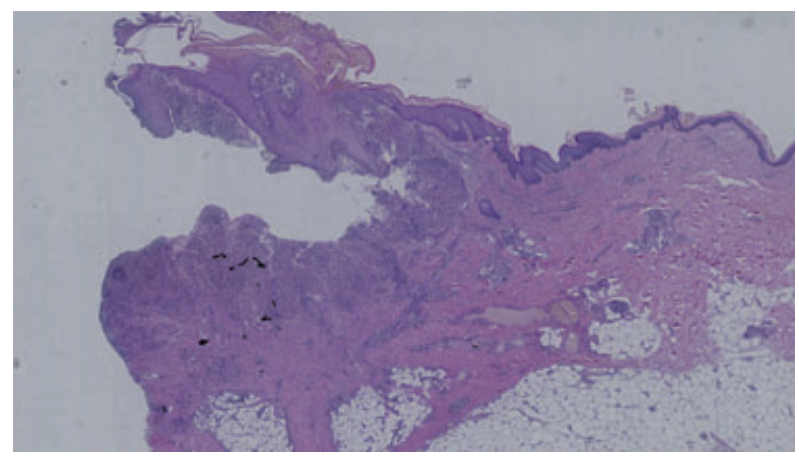

Figure 2 Skin biopsy showed diffuse non-necrotizing granulomatous inflammation with numerous multinucleated giant cells, lymphocytes, epithelioid cells, and plasma cell infiltration (hematoxylin-eosin $\times 40$ ) 